\title{
ESTUDIOS ECOLOGICOS DE CAMU-CAMU (Myrciaria dubia) PRODUCCION DE FRUTOS EN POBLACIONES NATURALES
}

$\left(^{*}\right)$ C. M. PETERS

(**) A. VASQUEZ

\section{RESUMEN}

En 1984 se inició un estudio a largo plazo sobre el crecimiento, reproducción y dinámica poblacional del camu-camu (M yrciaria dubia) en Sahua cocha, río U cayali. El presente artículo describe la biología floral de la especie y reporta dos años de datos de la producción de frutos. Las flores de M. dubia son hermafroditas pero muestran protógino durante la antesis que ayuda a evitar la autofecundación. Los polinizadores más importantes son dos especies de abejas (M elipona y Trigona). La producción de frutos fue altamente relacionada con el tamaño del individuo, siendo ambos efectuados en $1984\left(r^{2}=0.97\right)$ y $1985\left(r^{2}=0.99\right)$ y se calcula que las poblaciones naturales de la especie producen entre 9.5 y 12.7 toneladas de fruto/ha/año según las crecientes del río U cayali.

\section{SUM M ARY}

Long-term studies of the growth, reproduction and population dynamics of camu-camu (M yrciaria dubia) were initiated in 1984 at Sahua cocha, rio U cayali. the present contribution describes the floral biology of the species and reports two years of fruit production data. The flowers of $\mathrm{M}$. dubia are hermaphroditic but display protogyny during anthesis wich helps to reduce self fertilization. The primary pollinators are two species of bees (M elipona y Trigona). Fruit production was highly related with plant size both in $1984\left(r^{2}=0.97\right)$ and $1985\left(r^{2}=0.99\right)$. It is calculated that natural populations of the species produce between 9.5 and 12.7 tons of fruit/ha/yr depending on the fluctuations in the rio U cayali.

Palabras claves: camu-camu, M yrciaria dubia, biología floral, productividad, frutales nativos.

(*) Convenio Instituto de Investigaciones de la A mazonía Peruana e Instituto de Botánica Económica, Jardín Botánico de N ueva Y ork.

(**) Instituto de Investigaciones de la A mazonía Peruana. 


\section{INTRODUCCION}

Myrciaria dubia (HBK) Mc Vaugh, de la familia M yrtacea es un arbusto que crece comúnmente en la orilla de las quebradas y cochas de la cuenca amazónica. La especie forma una parte importante de la vegetación riparia en Perú, Brasil, Venezuela y Colombia, pero se presenta en mayor abundancia en la amazonía peruana, donde se encuentran poblaciones naturales extensas.

La planta es extremadamente tolerante a la inundación y puede quedar completamente sumergida en el agua durante cuatro o cinco meses. Reconocido por su corteza lisa y sus abundantes flores blancas, $\mathrm{M}$. dubia se conoce localmente como "camu-camu" en Perú o "cacari" en Brasil.

A demás del papel ecológico importante que tiene la especie, el camu-camu es un frutal nativo de gran potencial económico en la amazonía peruana. Como han señalado varios autores, su fruto contiene una alta concentración de ácido ascórbico $(2,000-2,994 \mathrm{mg} / \mathrm{l00} \mathrm{g})$ y representa una fuente rica de vitamina C (Ferreyra, 1959; Roca, 1965). El sabor agridulce del fruto es muy apreciado localmente y en la zona de Iquitos, donde se usa para hacer refrescos, helados y licores caseros. La gran mayoría de los frutos de camu-camu que abastecen el creciente mercado es cosechado de poblaciones naturales de la especie, algunas localizadas a mas de $150 \mathrm{Km}$. de Iquitos. Durante los últimos años esta especie ha despertado mucho interés y el numero de estudios relacionados con $M$. dubia ha aumentado notablemente. Se han establecido plantaciones en tierras no-inundables y numerosos trabajos han sido publicados sobre la propagación, enjertación y cultivo de camu-camu (Alvarado, 1969; Gutiérrez-Ruiz 1969; Pinedo, 1979; Calzada Benza, 1980). No obstante, se observa ausencia de información sobre la biología y productividad de camu-camu en su medio natural. L a fuente actual más grande de los frutos no ha sido evaluada.

Teniendo en cuenta esta situación, en 1984 se inició un estudio a largo plazo de la ecología de poblaciones naturales de M. dubia. El estudio está enfocado hacia el crecimiento, reproducción, mortalidad y dinámica poblacional de la especie, con el objeto de proporcionar bases ecológicas para el mejor manejo de este importante recurso.

El presente artículo, el primero en una serie, reporta dos años de datos de producción de frutos y describe la biología floral de M. dubia. Los otros resultados de la investigación serán presentados en futuras publicaciones relacionadas con germinación, establecimiento en el campo, crecimiento y ramificación, así como en la estabilidad ecológica de poblaciones naturales de M. dubia, sujeta a explotación intensiva. 


\section{MATERIALES Y METODOS}

Los estudios ecológicos de M. dubia se están llevando a cabo en Sahua cocha, en un sector del río U cayali presenta una superficie de $80 \mathrm{Km} ., 2$, que se encuentra a $6 \mathrm{Km}$. del pueblo de Jenaro Herrera; ubicado en la provincia de Requena, departamento de L oreto, Perú. La vegetación que rodea la cocha se caracteriza como bosque aluvial inundable o "seasonal varzea" en la clasificación de .Prance (1979). El clima de la zona es cálido-húmedo con una precipitación promedio anual do 288 $\mathrm{mm}$. y un promedio de temperatura de $25.9 \mathrm{C}$. Durante la creciente del río U cayali, el nivel de agua en Sahua Cocha puede subir hasta $12 \mathrm{~m}$. El camu-camu es muy abundante a lo largo de la orilla de la cocha y existe una área aproximada de 65 ha. de la especie.

En setiembre de 1984, se estableció una serie de cuadros contiguos de $10 \times 10$ $\mathrm{m}$. en un rodal denso de camu-camu, que estaba creciendo sobre el margen oeste de la cocha. Para determinar la densidad y estructura de la población, se midió el diámetro basal y se determinó la altura de todos los individuos de M. dubia en cada cuadro

Al mismo tiempo, se mapeó la posición exacta en el cuadro de cada planta, usando un sistema de coordenadas cartesianas y se enumeró con una etiqueta de metal. Se midió, también, toda la vegetación asociada de diámetro mayor de $1.0 \mathrm{~cm}$. (DAP) y se colectaron ejemplares para el herbario de cada especie, para su identificación. Debido al aumento constante del nivel de agua en la cocha, se tuvo que suspender el inventario después de completar 10 cuadros $01,000 \mathrm{~m}^{2}$ de muestreo.

El porcentaje de polinización y la tasa de aborto de frutos, fueron estimados periódicamente, mediante un submuestreo de ramas marcadas. Se seleccionaron al azar cinco ramas de cada uno de los 25 árboles, (representando un rango de diámetros basales diferentes) y se contaron todas las yemas florales y flores en cada rama. Durante todo el período reproductivo de los árboles, se registraron semanalmente número de frutos inmaduros y maduros. Antes de la inundación, el total de los frutos maduros fueron cosechados y contados.

En base al número total de frutos producidos, se calculó la producción total de flores y frutos inmaduros de cada rama, utilizando las tasas de polinización y aborto observadas en el submuestreo de ramas. El conteo de flores y frutos se continuaron oto los años 1984 y 1985.

Con el objeto de documentar el grado de autocompatibilidad que demuestra dubia, se efectuaron dos experimentos de polinización durante la floración de 
1984. En el primer experimento (una prueba de autogomia) se aislaron 15 flores con bolsas de malla fina, antes de la antésis, en cinco árboles diferentes. En el segundo experimento (una prueba de geitonogamia), se quitaron las antéras en tres repeticiones de 15 flores y se hizo polinización a mano con polen colectado de otras flores del mismo árbol previo a la colocación de las bolsas. Todas las flores fueron observadas por un mes y se registraron el número de fecundaciones obtenidas. Dado que el camu-camu está parcialmente inundado durante la fase reproductiva las investigaciones de floración y fructificación se tuvieron que hacer utilizando un bote.

Para estimar la producción anual de frutos en el área de estudio, primero sé realizó un análisis de regresión, utilizando como variables el diámetro basal y la fecundidad en los árboles muestreados durante 1984 y 1985. Debido a la naturaleza exponencial de la relación entre estas dos variables, se transformó a logaritmos los datos de fecundidad antes de hacer el análisis; asimismo, utilizando la ecuación obtenida de la regresión y la distribución diamétrica de los individuos, se calculó el número de frutos producidos por cada adulto en la población. Sumando todas las fecundidades individuales se estimó la productividad total.

\section{RESULTADOS}

\section{Dinámica de Población}

De las 14 especies de plantas leñosas encontradas en el área de estudio el camu-camu fue claramente el más dominante. Incluyendo plántulas (juveniles y adul. tos) la densidad de $M$. dubia fue de 1,231 individuos $/ 1000 \mathrm{~m}^{2}$, es decir, más de 12,000 individuos/ $\mathrm{Ha}$. Cabe destacar que esta densidad representa el número individuos genéticamente distintos o "genets" (sensu Harper, 1977) y no el número total de tallos 0 "ramets"

Esta distinción es importante, dada la profusa ramificación basal que demuestra la especie, porque cuando se incluyen los rebrotes, la densidad total de la población pasa a 1,500 tallo//000 $\mathrm{m}^{2}$. Datos detallados sobre la fitosociología del área de estudio se anexan en el A péndice I.

La distribución de los individuos de M. dubia por categoría de diámetro basal se presenta en la Figura 1. La distribución se ajusta bien a una curva exponencial, donde $r=0.92$, lo que refleja una característica de poblaciones de plantas estables que tienen la capacidad de mantenerse (M eyer 1952; Leak 1964). 
L a desviación de la curva en las categorías diamétrica pequeñas e intermedias, sugiere que la regeneración de la población no es constante, sino abundante en algunos años y limitada en otros.

Sin embargo, parece que la población de M. dubia logra una regeración significativa a través del tiempo, para compensar la mortalidad en las categorías diamétricas mayores.

\section{Biología Floral}

La mayoría de los individuos de camu-camu empiezan a florecer después de llegar a un diámetro basal de $2.0 \mathrm{~cm}$. La floración no está sincronizada en la copa de un individuo, pero ocurre en varios ciclos o pulsos. Las yemas florales brotan primero en la parte distal de las ramas mas altas y después de que se han abierto y ha pasado la polinización, otras yemas salen de un lugar mas proximal sobre la rama. De esta forma, se observa que la floración sigue desde las ramas de arriba hacia las de abajo y por lo tanto un individuo puede presentar yemas florales, así como flores y frutos en varios estados de desarrollo al mismo tiempo. Salen hasta 12 flores de cada nudo y se observó la formación de flores directamente del tronco y ramas gruesas de los individuos grandes.

L as flores individuales de M . dubia son hermafroditas, subsesiles, de 1.0 a 1.2 $\mathrm{cm}$. de diámetro, con cuatro pétalos blancos. Cada flor contiene un estilo de 8.0 a $9.2 \mathrm{~mm}$. de longitud y aproximadamente 125 estambres (Figura 2). L a antésis ocurre temprano en la mañana y las flores están receptibles para la polinización en un período de 4 a 5 horas. Después de la polinización los estambres empiezan a marchitarse y toda la corola seca se cae al día siguiente.

La emergencia del estilo y los estambres dentro de una flor, demuestra un protógino muy marcado (Figura 2). Durante la antésis, el estilo sale primero y después pasa un lapso de varias horas antes que salgan los estambres. L os resultados de los experimentos de polinización indican que este mecanismo es muy efectivo para evitar la autogamia. Tal como se presenta en Tabla 1, sólo una de las flores protegidas de fuentes externas de polen dió frutos. A parentemente, en el momento que salen los estambres para liberar polen el estigma ya no está receptible a la polinización.

La dicogamia que muestra M. dubia, sin embargo, no descarta la posibilidad de autofecundación por geitonogamia debida a la falta de sincronía floral. Polen de otras flores de la misma planta pueden todavía efectuar polinización; esto se probó en el segundo experimento de polinización, en el cual alto porcentaje $(91 \%)$ de flores 
92

polinizadas con polen de otras flores del mismo árbol formaron frutos (Tabla 1). Basado en estos resultados se puede concluir que el camu-camu presenta alogamía facultativa pero no obligatoria y no tiene mecanismos de incompatibilidad genética.

A unque un grado de polinización en camu-camu puede ser efectuado por el viento, los polinizadores mas importantes para la especie son las pequeñas abejas. L as flores contienen nectarios y exudan una fragancia dulce y agradable, por lo que en la mañana están cubiertas con abejas. Colecciones hechas en este estudio indican que Melipona fuscopilara y Trigona pórtica son los polinizadores mas comunes del camucamu en Sahua cocha.

\section{Fecundidad de los Individuos}

En Tabla 2 se presentan los datos promedios de producción de flores, frutos inmaduros y frutos maduros, correspondientes a los años 1984 y 1985.

Se agrupó los datos en categorías diamétricas de $2.0 \mathrm{~cm}$. y se calculó el porcentaje de polinización (número de frutos inmaduros/número de flores x 100), el porcentaje de abortos (número de frutos inmaduros-número de frutos maduros/número de frutos inmaduros $x$ 100) y el porcentaje de cuaje (número de frutos maduros/número de flores $x$ 100) por categoría. Como se puede apreciar en la tabla, con un incremento en el diámetro de los individuos la producción de flores, frutos inmaduros y frutos maduros aumenta en una forma exponencial.

El porcentaje de polinización y de cuaje se mantienen sorprendentemente constante, a través de todas las categorías, mientras que la taza de abortos para los individuos pequeños es significativamente más alta que para todos los demás $(\mathrm{P} \ll 0.01$, ANOVA con transformación de arcoseno). En términos generales el 46 por ciento de todas las flores de $\mathrm{M}$. dubia estan polinizadas y un promedio de 15 por ciento de los frutos inmaduros se abortan antes de llegar a la madurez.

Comparando los datos de fecundidad entre los dos años, se percibe que la mayoría de los árboles produjeron mas flores y frutos inmaduros en 1985 que en 1984 (P0.001, prueba de t). Sin embargo, los individuos de las dos últimas categorías diamétricas mostraron un patrón contrario con una mayor producción en 1984.

A pesar de los cambios en fecundidad, no se encontró ninguna diferencia significativa entre los porcentajes de polinización, aborto y cuaje en 1984 y 1985. 


\section{Producción T otal de Frutos}

Los resultados del análisis de regresión entre categorías de diámetro basal y producción de frutos en 1984 y 1985, se muestran en la Figura 3. Nótese que la gráfica es semi-logarítmica. La producción de frutos fue altamente relacionada con el tamaño del individuo en 1984 ( $r 2=0.97)$ y en 1985 ( $r 2=0.99)$, aunque la pendiente de la recta fue ligeramente diferente en cada año. Esta diferencia se debe al aumento en la producción de los individuos pequeños junto con la reducción en la fecundidad de los individuos grandes en 1985.

El cálculo de la producción total de frutos de camu-camu en Sahua cocha se presenta en la Tabla 3. Los datos del número de individuos por hectárea en cada categoría diamétrica se basa en el inventario del área de estudio. En ésta Tabla se puede observar que los rodales silvestres de M.dubia son extremadamente productivos, aunque los individuos grandes producen mas frutos por árbol, la mayor parte de la producción total proviene de las categorías diamétricas pequeñas, debido a la abundancia marcada de éstos individuos. Tomando en cuenta que Sahua cocha contiene un poco mas de 60 ha. de camu-camu, la capacidad total productiva de la cocha se estima entre 600 y 700 t/año, para lo cual se ha considerado que la producción total de frutos, calculada para 1984 y 1985 será equivalente a 9.5 y 12.7 t/ha., respectivamentes.

\section{DISCUSION}

L a mayor productividad de las poblaciones silvestres de M. dubia es probable que se deba al efecto del ambiente en cual crece la especie. A parentemente hay pocas especies de plantas leñosas toleran te a la inundación total, y por lo tanto, camu camu logra formar agrupaciones mono-específicas a lo largo de las cochas y quebradas. En la ausencia de competencia por otras plantas, el camu-camu puede aprovechar al máximo los altos niveles de radiación solar y la abundante humedad del suelo, característicos de su sitio ripario. El camu-camu también recibe un abono natural cada año con la creciente del río, cuando se depositan cantidades de sedimentos fértiles, por lo que no es tan sorprendente que la productividad de M. dubia en su medio natural sobrepase la cosecha obtenida de muchos frutales bajo cultivo intensivo.

Por otro lado, son las mismas fluctuaciones en el nivel de agua que delimitan el potencial productivo del camu-camu. Como indican los resultados de este estudio, la producción total de frutos está determinada más por el tiempo que la planta queda fuera del agua, que por la abundancia de polinizadores o la tasa de abortos. Por 
ejemplo, en 1984, el periódo de fructificación fue 27 días mas corto que el de 1985, debido a la creciente atrasada del río U cayali.

L as copas de camu-camu quedaron destapadas por mas tiempo, la producción de flores y frutos se prolongó y, como resultado, la producción total de frutos en ese año fue $30 \%$ superior a 1985 . A simismo, parece que cuando la creciente del río es relativamente lenta, los individuos pequeños son las que benefician. Estos individuos raramente tienen la oportunidad para mostrar todo su potencial reproductivo, porque siempre son los primeros en estar sumergidos. A unque no existe información publicada sobre las plantaciones de camu-camu que se han establecido en terrenos noinundables, datos proporcionados por Trigo- so y Pinedo (1985) y observaciones sobre las plantaciones experimentales del CLPA XVI en la estación de San Roque, cerca de Iquitos, indican que su productividad actual es relativamente baja, a pesar del uso de fertilizantes.

Sin mayor información, es difícil identificar la razón exacta para el bajo rendimiento, pero la falta de polinizadores o limitaciones de nutrientes son las causas mas probables. Sería interesante cuantificar el porcentaje de polinización y la tasa debortos de los individuos en plantaciones, para ver cual es el factor crítico. Dada la importancia del carnu -camu como frutal nativo y como fuente rica de vitamina $C$, el aprovechamiento de rodales naturales de la especie merece mucho mas atención. Son muy productivos y su uso no involucra gastos económicos altos 0 un tiempo de espera, mientras que las plantas llegan a la madurez. No cabe duda que la manera mas barata y eficiente de explotar el camu-camu es en su medio natural.

Sin embargo, para asegurar un rendimiento sostenido, es crítico que la explotación de poblaciones silvestres de M.dubia sea controlada. Además de tener un valor para el hombre, los frutos de camu -camu contienen las semillas necesarias para regenerar y mantener el rodal. Al mismo tiempo, los frutos representan un alimento importante para muchos peces de la región, como la gamitana (Colossoma. macropornurn, Fam. Characidae). La sobre explotación de poblaciones silvestres fácilmente puede tener un impacto negativo fuerte sobre la abundancia de M. dubia y sobre toda la cadena trófica, que está basada en sus frutos.

\section{REFERE NCIAS BIBLIOGRAFICAS}

ALVARAD0, M. 1969. Posibilidades del cultivo del camu-camu (M yrciaria dubia) en el Perú. Tesis Ingeniero A grónomo, Pont, U niv. Católica del Perú, Lima, $51 p$. 
CA LZA DDA-BENZA , J. 1980. 143 Frutales Nativos. Librería El Estudiante, Lima, $320 p$.

FERREYRA, R. 1959. Camu-carnu, nueva fuente nacional de vitamina C. Bol. Exp. A gropecuaria 7 (4): 28.

GUTIERREZ-RUIZ, A. 1969. Especies frutales nativas de la selva del Perú: estudio botánico y de propagación por semillas. Tesis Ingeniero A grónomo, Univ. Agr. La Molina, Lima, 105p.

HARPeR, J. L. 1977. Population Biology of Plants. A cademic Press, New York, $892 p$.

LEAK, W. B. 1964. An expression of diameter distribution for balanced, uneven-aged stands and forests. For. Sci. 10: 39-50.

MEYER, H. A. 1952. Structure, growth and dram in balanced even-aged forests. J. For. 50: 85-92.

PINEDO, M. 1979. Propagación de arazá (Eugenia stipitata) y camu-carnu (Myrciaria dubia). Tesis Ingeniero Agrónomo, Univ. Nac. Amazonía Peruana, Iquitos, 82p.

PRANCE, G.T. 1979. Notes on the vegetatión of Amazonia III. The terminology of Amazonian forest types subject to inundation. Brittonia 37 (1): 26-38.

ROCA, N.A, 1965. Estudio químico - bromatológico de la Myrciaria paraensis Berg. Tesis Química, Univ. Nac. Mayor San Marcos, Lima, 5 lp.

TRIGOSA-PINEDO, M. 1985. Determinación de la producción de frutos de camucamu (Myrciaria paraensis), Myrtaceae en Padre Isla, Iquitos, Perú. ineditado, $7 \mathrm{p}$. 


\section{FIGURA 1}

Distribución por categorías diamétricas de los individuos de M. Dubia en Sahua cocha. El número de individuos está graficado en una logarítmica

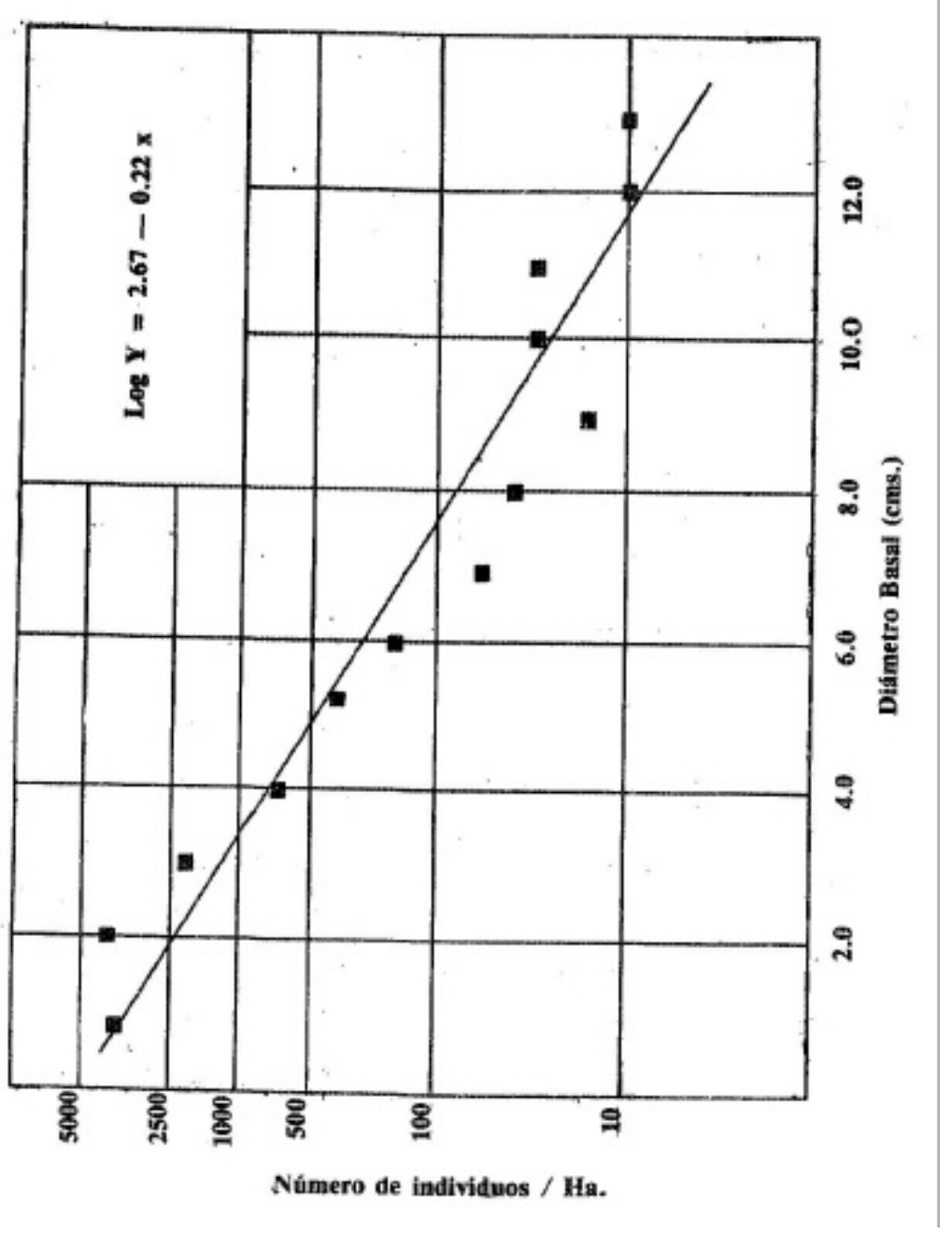




\section{FIGURA 2}

Desarrollo de las flores de M. Dubia.
(A y B) yema floral.
(C) E mergencia del estilo.
(D) E mergencia de los estambres.

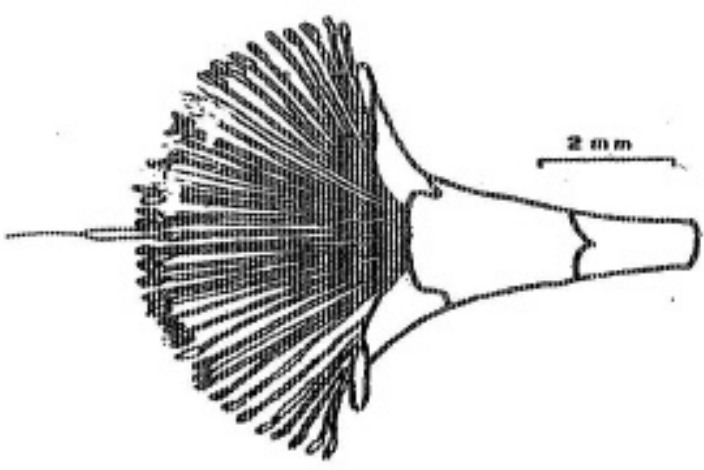

D

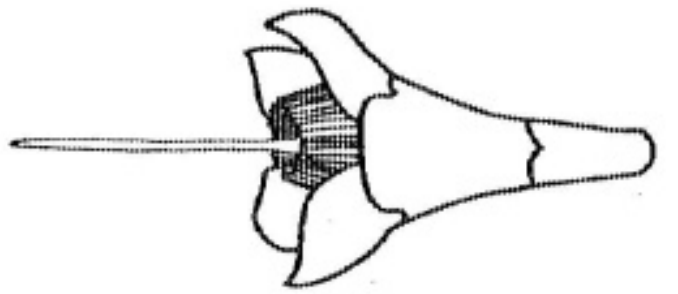

c

B

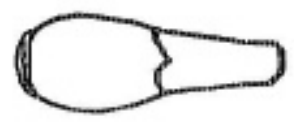

A 
98

TABLA 1 FORMACION DE FRUTOS EN M. DUbia DESPUES DE DOS TIPOS DE POLINIZACION CONTROLADA. CADA REPETICION REPRESENTA UN ARBOL DIFERENTE

\begin{tabular}{|c|c|c|}
\hline Experimento & Repetición & $\mathrm{N}$ \\
\hline
\end{tabular}

Prueba de autogamia

$\begin{array}{llll}\text { I } & 15 & 0 & 0.0 \\ \text { II } & 15 & 1 & 6.6 \\ \text { III } & 15 & 0 & 0.0\end{array}$

Prueba de geitonogamia

I

15

14

93.3

II

15

15

100.0

III

15

12

80.0 
TABLA 2 PRODUCCION DE FLORES Y FRUTOS INMADUROS POR M DUbIa EN SAHUA COCHA CON RELACION AL DIAMETRO BASAL. N ES IGUAL AL NUMERO DE FRUTOS DE ARBOL MUESTREADOS EN CADA CATEGORIA DIAMETRICA. VEASE EL TEXTO PARA EL CALCULO DE LOS PORCENTAJES DE POLINIZACION, ABORTO Y CUAJE.

\begin{tabular}{|c|c|c|c|c|c|c|c|c|}
\hline $\begin{array}{l}\text { Diámetro } \\
\text { basal }(\mathrm{cm}) \\
(\%)\end{array}$ & No & A ño & № Flores & $\begin{array}{c}\text { Polinización } \\
\%\end{array}$ & $\begin{array}{l}\mathrm{N} \cong \text { F rutos } \\
\quad \mathrm{nmm}\end{array}$ & $\begin{array}{l}\text { A bortos } \\
\text { aduros }\end{array}$ & $\begin{array}{c}\mathrm{N} N \text { - F rutos } \\
(\%)\end{array}$ & $\begin{array}{l}\text { Cuaje } \\
\text { M aduros }\end{array}$ \\
\hline \multirow[t]{2}{*}{$2.0-4.0$} & 5 & 1984 & $996.5 \pm 107.3$ & 48.3 & $481.3 \pm 51.1$ & 27.1 & $350.0 \pm 25.1$ & 35.2 \\
\hline & & 1985 & $1271.1 \pm 186.4$ & 46.7 & $560.4 \pm 93.7$ & 19.9 & $455.2 \pm 22.5$ & 37.4 \\
\hline \multirow[t]{2}{*}{$4.0-6.0$} & 5 & 1984 & $1359.2 \pm 149.5$ & 43.4 & $589.9 \pm 75.2$ & 10.8 & $526.8 \pm 25.3$ & 38.7 \\
\hline & & 1985 & $1861.9 \pm 167.6$ & 41.2 & $767.1 \pm 82.2$ & 12.1 & $674.9 \pm 37.9$ & 36.2 \\
\hline \multirow[t]{2}{*}{$6.0-8.0$} & 5 & 1984 & $1654.1 \pm 132.3$ & 47.3 & $782.4 \pm 117.4$ & 12.3 & $686.2 \pm 49.7$ & 41.5 \\
\hline & & 1985 & $2405.3 \pm 210.6$ & 45.8 & $1101.6 \pm 97.7$ & 13.5 & $952.5 \pm 110.8$ & 39.6 \\
\hline \multirow[t]{2}{*}{$8.0-10.0$} & 4 & 1984 & $3642.0 \pm 582.7$ & 45.0 & $1638.5 \pm 229.4$ & 15.6 & $1383.3 \pm 105.7$ & 38.0 \\
\hline & & 1985 & $3586.2 \pm 346.0$ & 47.1 & $1689.1 \pm 381.7$ & 13.8 & $1456.0 \pm 178.0$ & 40.6 \\
\hline \multirow[t]{2}{*}{$10.0-12.0$} & 3 & 1984 & $6144.2 \pm 675.9$ & 48.6 & $2906.1 \pm 268.7$ & 13.7 & $2577.0 \pm 201.0$ & 41.9 \\
\hline & & 1985 & $5461.1 \pm 613.5$ & 46.3 & $2528.5 \pm 233.6$ & 14.4 & $2162.6 \pm 230.9$ & 39.6 \\
\hline \multirow[t]{2}{*}{$12.0-14.0$} & 3 & 1984 & $9398.3 \pm 1127.8$ & $8 \quad 48.3$ & $4539.4 \pm 441.5$ & 12.4 & $3975.5 \pm 413.2$ & 42.3 \\
\hline & & 1985 & $7804.3 \pm 926.5$ & 49.5 & $3863.1 \pm 367.3$ & 11.7 & $3410.5 \pm 570.5$ & 43.7 \\
\hline
\end{tabular}




\section{FIGURA 3}

Relación entre el diámetro basal y producción de frutos 1984 y 1985 por M. dubia en A súa cocha. L os datos de producción de frutos están graficados en una escala logarítmica.

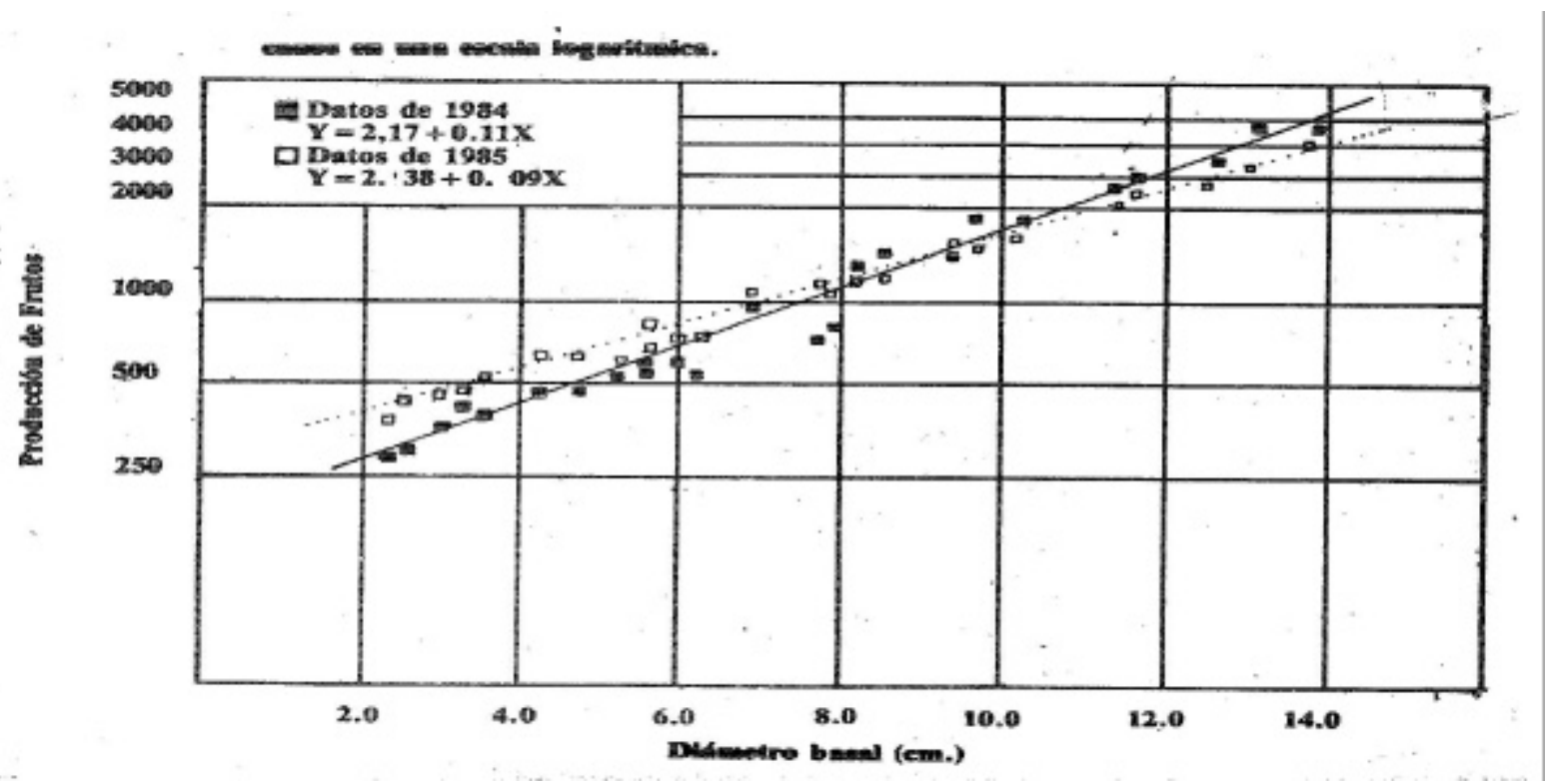


TABLA 3 PRODUCCION TOTAL ANUAL DE FRUTOS POR M Dubia EN SAHUA COCHA CALCULADAS PARA CATEGORIAS DIAMETRICAS Y PARA LA TODA LA POBLACION

\begin{tabular}{|c|c|c|c|c|c|}
\hline \multirow[b]{2}{*}{$\begin{array}{c}\text { Diámetro basal } \\
(\mathrm{cm})\end{array}$} & \multicolumn{3}{|c|}{ Producción 1984} & \multicolumn{2}{|c|}{1985} \\
\hline & Individuos/Ha. & $\begin{array}{r}\text { Producción } \\
\text { de frutos } \\
\end{array}$ & $\%$ Total & $\begin{array}{l}\text { Producción } \\
\text { de frutos }\end{array}$ & $\%$ T otal \\
\hline $2.0-3.0$ & 1800 & 506,731 & 42.0 & 744,627 & 46.2 \\
\hline $3.0-4.0$ & 600 & 214,727 & 17.8 & 301,213 & 18.7 \\
\hline $4.0-5.0$ & 310 & 141,035 & 11.7 & 189,682 & 11.8 \\
\hline $5.0-6.0$ & 150 & 86,753 & 7.3 & 107,010 & 6.7 \\
\hline $6.0-7.0$ & 50 & 36,761 & 3.0 & 43,548 & 2.7 \\
\hline $7.0-8.0$ & 40 & 37,386 & 3.1 & 42,860 & 2.6 \\
\hline $8.0-9.0$ & 20 & 23,763 & 1.9 & 25,963 & 1.6 \\
\hline $9.0-10.0$ & 30 & 45,315 & 3.7 & 47,546 & 2.9 \\
\hline $10.0-11.0$ & 30 & 57,606 & 4.8 & 58,948 & 3.7 \\
\hline $11.0-12.0$ & 10 & 24,410 & 2.1 & 27,754 & 1.7 \\
\hline $12.0-13.0$ & 10 & 31,032 & 2.6 & 27,754 & 1.7 \\
\hline Producción tota & anual & $1^{\prime} 205,519$ & & $1^{\prime} 616,905$ & \\
\hline
\end{tabular}


102

APENDICE 1 COMPOSICION FLORISTICA DEL AREA DE ESTUDIO (SAHUA COCHA, RIO UCAYALI ESTAN INCLUIDAS TODAS LAS PLANTAS CON DIAMETRO (DAP) MAS GRANDE QUE 1.0 CMS.; EJEMPLARES DE ELLOS ESTAN DEPOSITADAS EN LOS HERVARIOS DEL CENTRO DE INVESTIGACIONES DE JENARO HERRERA Y DEL NEW YORK BOTANICAL GARDEN (NY)

\begin{tabular}{|c|c|c|c|c|}
\hline Especies & $\begin{array}{l}\text { Densidad } \\
\text { No. } 1000 \mathrm{~m}^{2}\end{array}$ & $\begin{array}{l}\text { F recuencia } \\
(\%)\end{array}$ & $\begin{array}{l}\text { A rea Basal } \\
\left(\mathrm{cm}^{2}\right)\end{array}$ & V.I \\
\hline M yrciaria dubia (HBK) M cV augh & 1231 & 40 & 7614.2 & 84.0 \\
\hline Eugenia inundata DC & 226 & 35 & 2339.9 & 41.4 \\
\hline Couepia sp. & 16 & 9 & 172.3 & 8.4 \\
\hline$L$ aetia americana $L$. & 16 & 7 & 39.1 & 6.3 \\
\hline Symmeria paniculada Bent. & 11 & 5 & 336.5 & 5.4 \\
\hline Triplaris peruviana Fish. Et M ey & 5 & 3 & 14.5 & 2.6 \\
\hline Alchornea sp. & 3 & 3 & 9.3 & 2.6 \\
\hline M ouriri grandiflora DC. & 3 & 3 & 6.6 & 2.6 \\
\hline L uchea tessmammii Burret. (Benth) Benth & 3 & 3 & 6.3 & 2.6 \\
\hline M acrolobium acaciaefolium & 2 & 2 & 101.7 & 2.0 \\
\hline Eugenia patrissi $V$ ahl. & 2 & 2 & 17.3 & 1.7 \\
\hline C rataeva tapia L & 2 & 2 & 7.2 & 1.7 \\
\hline Cecropia sp. & 1 & 1 & 17.3 & 0.9 \\
\hline M argaritaria nobilis L. & 1 & 1 & 2.8 & 0.9 \\
\hline
\end{tabular}

Valor de importancia $=$ suma de densidad relativa, frecuencia relativa y área basal relativa dividida por tres. 